\title{
Antitumor and apoptosis-promoting properties of emodin, an anthraquinone derivative from Rheum officinale Baill, against pancreatic cancer in mice via inhibition of Akt activation
}

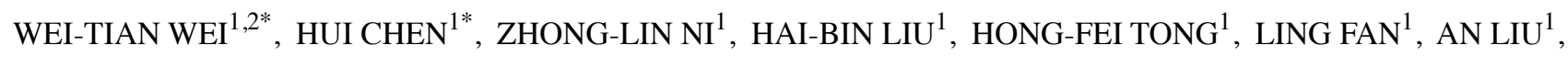 \\ MAI-XUAN QIU ${ }^{1}$, DIAN-LEI LIU ${ }^{1}$, HONG-CHUN GUO ${ }^{1}$, ZHAO-HONG WANG ${ }^{1}$ and SHENG-ZHANG LIN ${ }^{1}$ \\ ${ }^{1}$ Department of Hepato-biliary-pancreatic Surgery, The Second Affiliated Hospital of Wenzhou Medical College, No. 109, \\ West Xue-yuan Road, Wenzhou 325027; ${ }^{2}$ Department of Oncological Surgery, Zhejiang Cancer Hospital, P.R. China
}

Received February 1, 2011; Accepted April 8, 2011

DOI: 10.3892/ijo.2011.1147

\begin{abstract}
Pancreatic adenocarcinoma is one of the most common malignancies worldwide. Gemcitabine is currently the standard first-line chemotherapeutic agent for pancreatic cancer. However, gemcitabine can induce activation of Akt and nuclear factor- $\kappa \mathrm{B}$ $(\mathrm{NF}-\mathrm{kB})$, which is associated with its chemoresistance. It has been reported that gemcitabine combination therapies result in improved survival outcomes in pancreatic cancer. Therefore, agents that can either enhance the effects of gemcitabine or overcome chemoresistance to the drug are needed for the treatment of pancreatic cancer. Emodin is an active component of Chinese medicinal herbs and can inhibit the activation of Akt and NF- $\kappa B$. In this study, we investigated whether emodin could enhance the anticancer effect of gemcitabine on pancreatic cancer in vivo. We demonstrated that treatment of gemcitabine combined with emodin efficiently suppressed tumor growth in mice inoculated with pancreatic tumor cells. This treatment paradigm promoted apoptotic cell death and mitochondrial fragmentation. Furthermore, it reduced phosphorylated-Akt (p-Akt) level, NF- $\kappa$ B activation and Bcl-2/Bax ratio, increased caspase-9 and -3 activation, Cytochrome $\mathrm{C}(\mathrm{CytC})$ release occurred in combination therapy. Collectively, emodin enhanced the activity of gemcitabine in tumor growth suppression via inhibition of Akt and NF- $\mathrm{KB}$ activation, thus promoting the mitochondrial-dependent apoptotic pathway. Therefore, our findings may provide new insights into understanding the pharmacological regulation of emodin on gemcitabine-mediated proapoptosis in pancreatic
\end{abstract}

Correspondence to: Dr Sheng-Zhang Lin, The Second Affiliated Hospital of Wenzhou Medical College, No. 109, West Xue-yuan Road, Wenzhou 325027, P.R. China

E-mail:wzf21sz@163.com

${ }^{*}$ Contributed equally

Key words: emodin, gemcitabine, SW1990 cells, apoptosis, p-Akt, $\mathrm{NF}-\kappa \mathrm{B}$ cancer and may aid in the design of new therapeutic strategies for the intervention of human pancreatic cancers.

\section{Introduction}

Pancreatic cancer is a relatively uncommon but highly aggressive and lethal malignancy with a very low median survival, which is correctly the perception from the general population $(1,2)$. Surgery is believed to be the only prospective cure, although the resection rate is relatively low (3). This is at least partially due to the fact that only $10-15 \%$ of pancreatic adenocarcinoma patients are candidates for surgery due to the asymptomatic nature of early stage pancreatic cancer (4).

The use of chemotherapy in the treatment of pancreatic cancer has been receiving considerable attention lately. A variety of compounds targeting various intracellular survival pathways have been widely studied, including antimetabolites, nucleoside cytidine analogues and platinum analogues (5). Among these novel molecules, gemcitabine (2',2'-difuorodeoxycytidine), an S-phase nucleoside analogue, has shown significant clinical potential in treating various solid tumors, including pancreatic cancer (5). For advanced pancreatic cancer, patients treated with gemcitabine exhibited a 5-11\% response rate and a median survival rate of 5.7-6.3 months in phase I and II studies $(6,7)$. Moreover, combination treatment consisting of gemcitabine and 5-fluorouracil (5-FU) (8), oxaliplatin (9), cisplatin (10) or irinotecan (11) has also resulted in improved responses in patients with advanced pancreatic cancer. Although numerous doublet or triplet gemcitabine combination therapies have been shown to provide modest survival benefits compared to monotherapy, the response and median survival in patients with advanced pancreatic cancer do not increase (12). Therefore, development of novel drugs or therapies with high antitumor activity, commercial availability, low cost and low toxicity is imperative.

Emodin (1,3,8-trihydroxy-6-methylanthraquinone), an active component in Chinese herbs, including Rheum ofcinale and Polygonum cuspidatum, has been proven to have anti-cancer, anti-bacterial, anti-inflammatory, diuretic, immunosuppressive and vasorelaxant properties (13-16). Recent work has shown that emodin can suppress the proliferation of cancer cells, and induce apoptosis through a reactive oxygen species (ROS)-dependent 
mitochondrial signaling pathway $(17,18)$. Furthermore, a recent report showed that emodin promoted pancreatic cancer cell apoptosis induced by gemcitabine via inhibiting survivin (a member of the inhibitor of apoptosis gene family) expression (19). However, the mechanism by which gemcitabine plus emodin combination treatment induces pancreatic cancer cell apoptosis is still poorly understood.

In this study, we investigated the efficiency of emodin and gemcitabine combination treatment in vivo tumor growth inhibition and further examined the mechanism of combination therapy-induced pancreatic cancer cell apoptosis. Our results demonstrated that the combination treatment promoted apoptotic cell death in pancreatic tumor cells via inactivation of $\mathrm{Akt}$ and $\mathrm{NF}-\mathrm{kB}$, and subsequently a reduction in the $\mathrm{Bcl}-2 / \mathrm{Bax}$ ratio, enhanced Cytochrome $\mathrm{C}(\mathrm{CytC})$ release, and increased caspase- 9 and -3 activation.

\section{Materials and methods}

Reagents. Emodin was purchased from Sigma-Aldrich (St. Louis, MO, USA) and dissolved in dimethyl sulfoxide (DMSO). Gemcitabine was obtained from Lilly, France. D-luciferin potassium salt (Xenogen, Hopkinton, MA) was dissolved in sterile PBS at $40 \mathrm{mg} / \mathrm{ml}$ concentration. Rabbit anti-human, anti- $\beta$-actin, anti-Bax, and anti-bcl-2 polyclonal antibodies, as well as anti-CytC, anti-active caspase-9, anti-active caspase-3, and anti-NF-kB (p65) monoclonal antibodies were purchased from Abcam. Anti-phospho-Akt (Ser473) (phosphorylated Akt, p-Akt) monoclonal antibody was purchased from Cell Signaling (Beverly, MA). Anti-retinoblastoma antibody was from Santa Cruz Biotechnology (Santa Cruz, CA). TdT-mediated dUTP nick-end labeling (TUNEL) staining kit was purchased from Roche (Germany).

Cell culture. The human pancreatic adenocarcinoma cell line SW1990 was obtained from American Type Culture Collection (ATCC, USA). SW1990 cells were stably transfected with luciferase as previously described for Panc-1 cells (20). Luciferase-transfected SW1990 cells were routinely cultured in Roswell Park Memorial Institute 1640 (RPMI-1640) culture medium (Gibco) supplemented with $10 \%$ fetal bovine serum (FBS), penicillin $(100 \mathrm{U} / \mathrm{ml})$ and streptomycin $(100 \mathrm{U} / \mathrm{ml})$. Cells were maintained in a humidified atmosphere of $5 \% \mathrm{CO}_{2}$ at $37^{\circ} \mathrm{C}$. Cells were passaged at $70-80 \%$ confluence. Luciferasetransfected SW1990 cells were harvested from 70-80\% confluence cultures after a brief exposure to $0.25 \%$ trypsin and $0.2 \%$ EDTA. Trypsinization was stopped with medium containing $10 \%$ FBS. The cells were washed once in serum-free medium and resuspended in PBS. Only suspensions consisting of single cells, with $>90 \%$ viability, were used for the injections.

Pancreatic cancer xenograft tumor model. Luciferase-transfected SW1990 cells in log-phase were digested with trypsin and resuspended in serum-free culture medium. Tumor xenografts were established by subcutaneous inoculation of $2 \times 10^{6}$ luciferasetransfected SW1990 pancreatic cancer cells into the right abdominal flanks of BALB/c female nude mice (4-6 weeks old, 18-20 g in weight, purchased from Shanghai Laboratory Animal Center, Chinese Academy of Sciences, Shanghai, China). After 2 weeks of implantation, a total of 48 nude mice were randomized into four treatment groups $(\mathrm{n}=12)$ based on the bioluminescence measured after the first IVIS imaging, 12 mice in each group, and housed in a specific pathogen-free (SPF) laboratory. Three weeks after inoculation, when tumors had grown to the desired size (largest diameter $\geq 5 \mathrm{~mm}$ ), the mice received different treatments every three days. Animals in the emodin group, gemcitabine group, emodin and gemcitabine combination therapy group or control group received intraperitoneal injections of $40 \mathrm{mg} / \mathrm{kg}$ emodin, $125 \mathrm{mg} / \mathrm{kg}$ gemcitabine, $40 \mathrm{mg} / \mathrm{kg}$ emodin plus $80 \mathrm{mg} /$ $\mathrm{kg}$ gemcitabine, and $0.9 \%$ sodium chloride, respectively. Mouse body weight were measured by an electronic balance and tumor size were measured by a vernier caliper every six days. Tumor size was calculated using the following formula: Volume $=(4 / 3) \pi$ $[(\text { length }+ \text { width }) / 4]^{3}$. The tumor growth inhibition ratio was calculated using the following formula: Inhibition rate $(\%)=$ [1 - (mean tumor volume of drug-treated mice before experiment - mean tumor volume of drug-treated mice at the end of the experiment)/(mean tumor volume of control mice before experiment - mean tumor volume of control mice at the end of the experiment)] x $100 \%$. At the meantime, tumor volumes were monitored every six days by the bioluminescence IVIS Imaging System 200 using a cryogenically cooled imaging system coupled to a data acquisition computer running Living Image software (Xenogen Corp., Alameda, CA). Before imaging, animals were anesthetized in an acrylic chamber with $2.5 \%$ isoflurane/air mixture and injected i.p. with $40 \mathrm{mg} / \mathrm{ml}$ D-luciferin potassium salt in PBS at a dose of $150 \mathrm{mg} / \mathrm{kg}$ body weight. After $10 \mathrm{~min}$ of incubation with luciferin, mice were placed in a right lateral decubitus position and a digital grayscale animal image was acquired followed by acquisition and overlay of a pseudocolor image representing the spatial distribution of detected photons emerging from active luciferase within the animal. Signal intensity was quantified as the sum of all detected photons within the region of interest per second. Therapy was continued for 30 days and animals were sacrificed one week after the final injection (day 37). The final tumor volume was measured for last time by a vernier caliper and IVIS Imaging System before subcutaneous tumors were excised. A small part of tumor tissue was used for transmission electron microscopy (TEM) analysis. A part of the tumor tissue was formalin-fixed and paraffinembedded for immunohistochemistry, tunel assay and routine $\mathrm{H} \& \mathrm{E}$ staining. The other part was snap-frozen in liquid nitrogen. $\mathrm{H} \& \mathrm{E}$ staining confirmed the presence of subcutaneous pancreatic cancer. Ethical approval for this study was given by the Ethics Committee at Wenzhou Medical College.

TdT-mediated dUTP-biotin nick-end labeling (TUNEL) assay. One week after the final drug therapy, mice were anaesthetized with chloral hydrate. Tumor tissues were fixed in $4 \%$ formalin, embedded in paraffin, deparaffinized, and sectioned into $4 \mu \mathrm{m}$ serial sections. TUNEL technique was performed according to the manufacturer's instructions. Briefly, specimens were incubated for $1 \mathrm{~h}$ at $37^{\circ} \mathrm{C}$ in a moist chamber with TUNEL solution. TUNEL signals were visualized under a laser scanning confocal imaging system (FV1000 + SIMS, Olympus, Japan). At least 10 fields were randomly selected from each tumor section.

Immunohistochemical analysis. Formalin-fixed, paraffinembedded tumor tissues were sectioned and blocked with goat serum and immunostained after deparaffinization and rehydra- 
tion. Sections were incubated with anti-human anti-Bax, -Bcl-2, -CytC, or -p-Akt primary antibodies in a moist chamber overnight at $4^{\circ} \mathrm{C}$. After phosphate-buffered saline (PBS) rinses, specimens were incubated for another $30 \mathrm{~min}$ at room temperature with horseradish peroxidase (HRP)-conjugated secondary antibody. After staining with hematoxylin, sections were mounted and evaluated via microscopy (Olympus, Japan). Non-specific primary antibody staining was evaluated by substitution of the primary antibody with PBS. At least 6 fields were randomly selected from each section. Images were analyzed by Image-Pro Plus 6.0 software.

Western blotting. Proteins were routinely extracted from tumor tissues using radioimmunoprecipitation assay (RIPA) buffer. To determine Cytosolic CytC concentration, mitochondrial and cytosolic proteins were separated. Briefly, tissue lysates were prepared from mouse tumors and centrifuged at $800 \mathrm{x} \mathrm{g}$ for $5 \mathrm{~min}$ at $4^{\circ} \mathrm{C}$. The supernatant fraction of each sample was collected and centrifuged again. Fresh supernatant was transferred to a new tube and centrifuged at $12,000 \mathrm{x}$ g for $10 \mathrm{~min}$ at $4^{\circ} \mathrm{C}$. Supernatant containing cytosolic protein was collected and stored at $-80^{\circ} \mathrm{C}$ until use, and the pellet consisted of mitochondrial protein. Protein concentration was determined by bicinchoninic acid (BCA) assay. For Western blotting, $80 \mu \mathrm{g}$ of each sample was separated by $12 \%$ sodium dodecyl sulfatepolyacrylamide gel electrophoresis (SDS-PAGE), transferred to a polyvinylidene difluoride (PVDF) membrane (Millipore, USA), blocked with 5\% non-fat milk, and probed with primary antibodies followed by HRP-conjugated anti-rabbit secondary antibody. After washing, the bound antibody complexes were detected using an enhanced chemiluminescence (ECL) reagent (Amersham). Band images were analyzed by TotalLab 2.1 software.

Electrophoretic mobility shift assay (EMSA). NF- $\kappa \mathrm{B}$ activity was evaluated by EMSA analysis. Nuclear extraction was carried out according to the manufacturer's instructions (Pierce), and protein concentration was determined by BCA assay. The EMSA assay is based on that DNA-protein complexes migrate slower than unbound DNA or double-stranded oligonucleotides in a native polyacrylamide or agarose gel, resulting in a shift in migration of the labeled DNA band. The detection of bands was by The LightShift ${ }^{\mathrm{TM}}$ Chemiluminescent EMSA kit (Pierce) that uses a non-isotopic method to detect DNA-protein interactions. Biotin end-labeled DNA duplex of sequence 5'-AGT TGA GGG GAC TTT CCC AGG C-3' and 3'-TCA ACT CCC CTG AAA GGG TCC G-5' containing a putative binding site for nuclear factor- $\kappa \mathrm{B}$ was incubated with the nuclear extracts. After the reaction the DNA-protein complexes were subjected to a $6 \%$ native polyacrylamide gel electrophoresis and transferred to a nylon membrane (Biodyne B membrane, Pierce). After transfer the membrane was immediately cross-linked for $15 \mathrm{~min}$ on a UV transilluminator equiped with $312 \mathrm{~nm}$ bulbs. A chemiluminescent detection method utilizing a luminol/enhancer solution and a stable peroxide solution (Pierce) was used as described by the manufacturer, and membranes were exposed to X-ray film for 2-5 min before developing. The bands were scanned with an Epson Expression 1600 Pro, and relative intensities were analyzed by using an NIH Image 1.62 package. As for gel shift controls, nuclear extracts from gastric cancer SGC7901 was used without any treatment as negative control and from that stimulated with $50 \mathrm{ng} / \mathrm{ml} \mathrm{TNF} \alpha$ was used as positive control.

Transmission electron microscopy (TEM) analysis. Fresh tissue samples were fixed in $2.5 \%$ glutaraldehyde solution for $2 \mathrm{~h}$ and post-fixed in $1 \% \mathrm{OsO}_{4}$ solution for $1 \mathrm{~h}$. After dehydration, samples were embedded in Epon-812. Ultrathin sections (70 nm) were stained with uranyl acetate and lead citrate and examined using an H-7800 TEM (Hitachi, USA).

Statistical analysis. Data were analyzed using SPSS 17.0 software and plotted as mean \pm SD. Statistically significant differences were determined by one-way analysis of variance (ANOVA). $\mathrm{P}<0.05$ or $<0.01$ indicated a significant difference.

\section{Results}

Effects of combination treatment on mouse body weight and tumor size. The schematic overview of the study protocol is illustrated in Fig. 1A. A total of 48 mice were inoculated with SW1990 pancreatic cancer cells and randomly assigned to 4 experimental groups. Three weeks after inoculation, the average body weight was $21.2 \pm 0.8 \mathrm{~g}$ and the average tumor volume was $0.44 \pm 0.11 \mathrm{~cm}^{3}$, and no differences were observed among the groups $(\mathrm{P}>0.05)$. Animals then received intraperitoneal injections of $0.9 \%$ sodium chloride, emodin, gemcitabine, or emodin plus gemcitabine. We found that the body weight of mice in each group gradually decreased. One week after the final injection (day 37), the body weight in control group, emodin therapy group, gemcitabine group and combination therapy group was $16.6 \pm 1.5,17.0 \pm 1.7,13.0 \pm 1.8$, and $15.9 \pm 1.5 \mathrm{~g}$, respectively. A significant reduction in body weight was detected in the gemcitabine treatment group compared to other groups $(\mathrm{P}<0.05)$. Emodin, gemcitabine, and emodin plus gemcitabine treatments dramatically suppressed tumor growth, and combination drug therapy was more effective in tumor growth inhibition than monotherapy with either agent as measured by vernier caliper and bioluminescence IVIS Imaging (Fig. 1B-E). One week after the final injection (day 37), the final tumor growth inhibition rate in emodin group, gemcitabine group and combination group was $71.3,83.3$, and $103.7 \%$, respectively, while a highest tumor growth inhibition rate was measured in combination group compared to emodin or gemcitabine single agent group. These results showed that emodin plus gemcitabine combination treatment efficiently suppressed tumor growth in pancreatic tumor cell inoculated mice.

Combination treatment promoted apoptotic cell death in pancreatic tumor cells. Apoptosis is a major pathway leading to tumor cell death. Therefore, we investigated apoptotic cell death induced by drug treatment. As shown in Fig. 2A, an increased number of TUNEL-positive cells was observed in drug therapy groups, and combination treatment significantly promoted apoptotic cell death. Quantification of the data revealed that combination therapy consisting of emodin plus gemcitabine dramatically elevated integrated optical density (IOD) when compared with other groups $(\mathrm{P}<0.01)$ (Fig. 2B). According to electron microscopy analysis, among all organelles, mitochondria were likely the first to be attacked by drug treatment. Mitochondria were rod-like, with a dark and uniform matrix, 


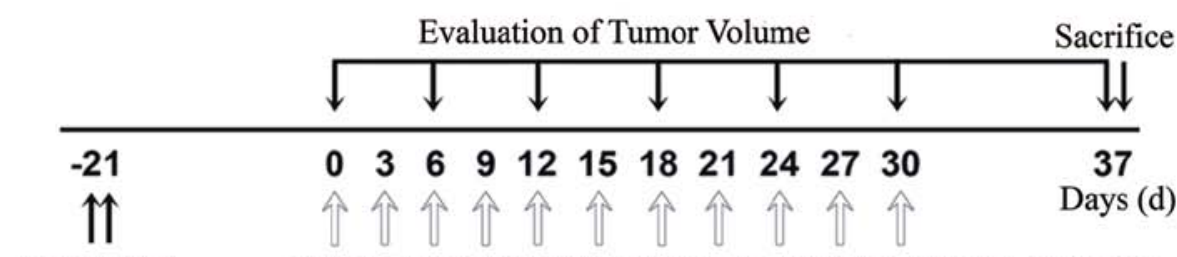

Tumor Cell

Intraperitoneal Injection of Sodium Chloride, Emodin $40 \mathrm{mg} / \mathrm{kg}$,

Inoculation Gemcitabine $125 \mathrm{mg} / \mathrm{kg}$ and Emodin $40 \mathrm{mg} / \mathrm{kg}+\mathrm{Gemcitabine} 80 \mathrm{mg} / \mathrm{kg}$

(A)
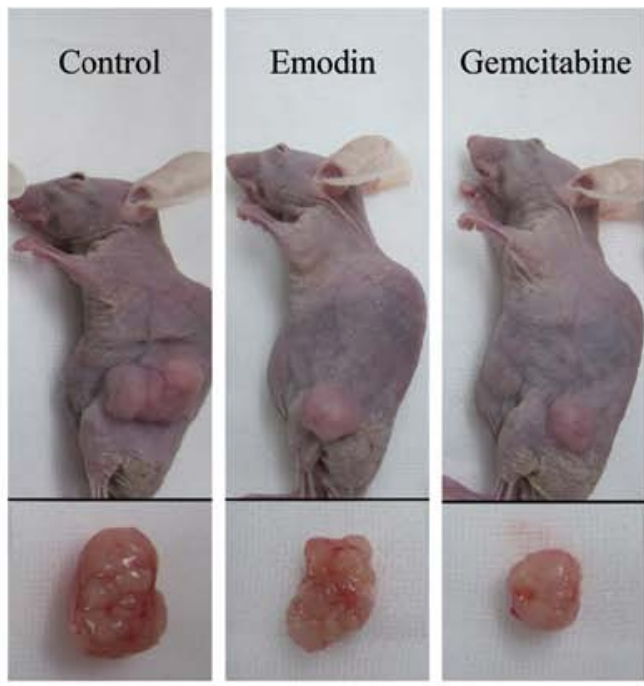

(B)

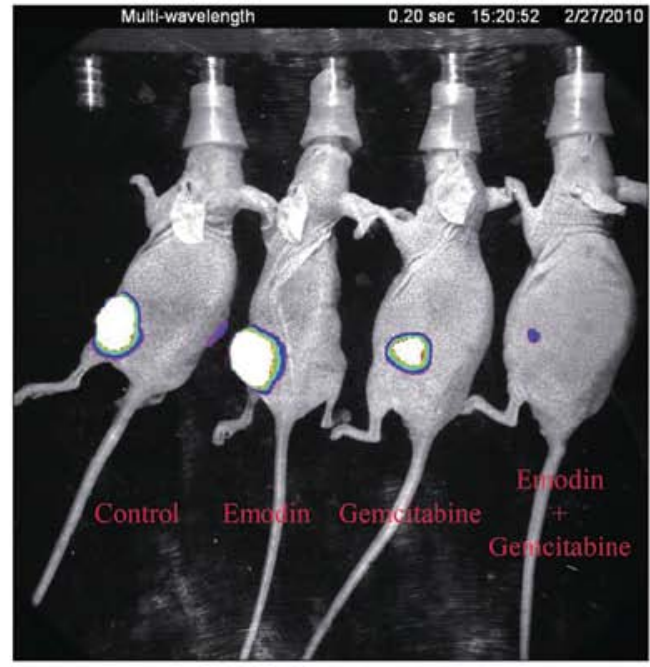

(D)

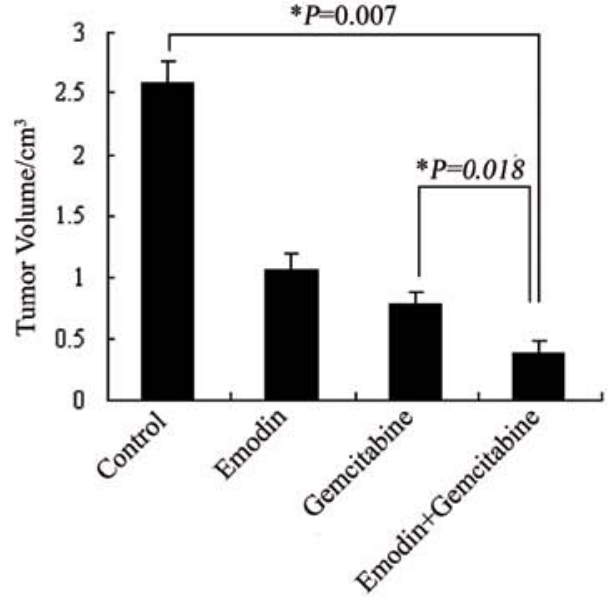

(C)

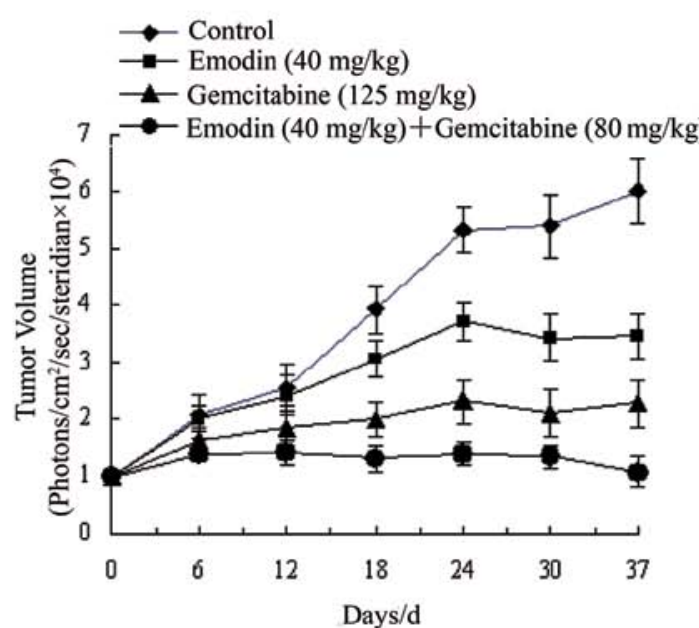

(E)

Figure 1. The effect of treatment on tumor size. (A) Schematic overview of the study protocol. (B) Photographs of mice bearing subcutaneously implanted pancreatic tumors and the removed tumors. (C) Tumor volumes in mice measured on the last day of the experiment (day 37) at biopsy using Vernier calipers and calculated using the formula: Volume $=(4 / 3) \pi[(\text { length }+ \text { width }) / 4]^{3}(n=12)$. (D) Bioluminescence IVIS images of subcutaneously implanted pancreatic tumors in live, anesthetized mice. (E) Measurements of photons per second depicting tumor volume at various time-points using live IVIS imaging at the indicated days $(\mathrm{n}=12)$. Points, mean; bars, SD.

and with regularly distributed cristae in control-treated tumor cells (Fig. 2C, arrows). However, in drug-treated groups, a large number of the mitochondria were small and round structures with few, if any, remaining cristae that were morphologically normal. In the tumor tissues of animals receiving combination therapy, fragmented mitochondrial membranes were detected. These results suggested that mitochondria-related pathways may be involved in combination therapy-induced apoptotic cell death.

Combination treatment reduced Akt activation. The serine/threonine kinase Akt, also known as protein kinase B (PKB), is the target of phosphatidylinositol 3-kinase (PI3K). Akt can induce activation of NF- $\mathrm{KB}$ and promote cell survival (21). Therefore, 

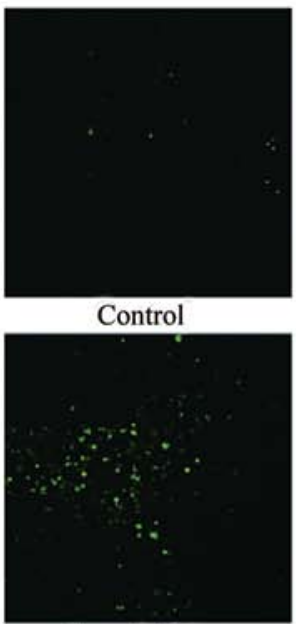

Gemcitabine

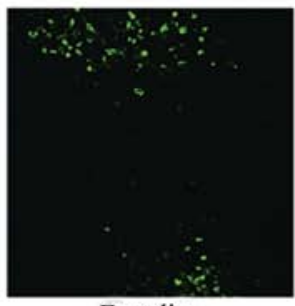

Emodin

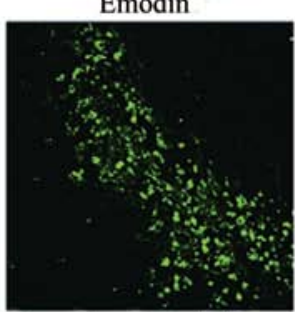

(A)

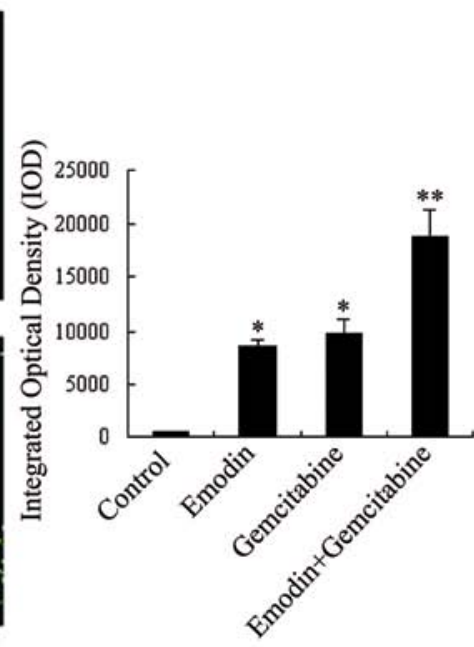

(B)

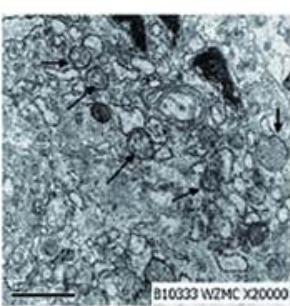

Control

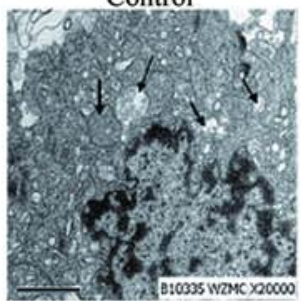

Gemcitabine

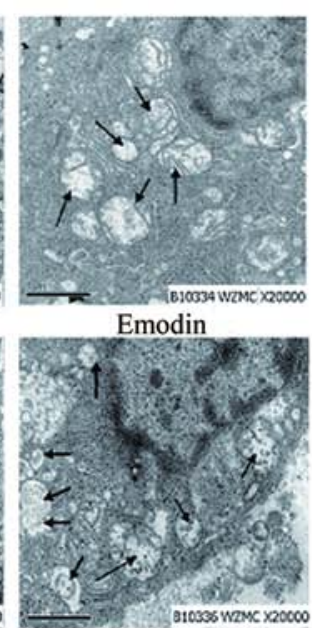

(C)

Figure 2. Combination treatment promoted apoptotic tumor cell death. (A) Tumor cell apoptosis was evaluated by TUNEL assay. Increased TUNEL-positive cells were found in the combination treatment group (x400). (B) TUNEL staining was further quantified and presented as average IOD level. * $<0.01$ compared to sodium chloride treatment; ${ }^{* *} \mathrm{P}<0.01$ compared to sodium chloride, emodin or gemcitabine treatment. (C) Ultrastructure of tumor tissues showed by TEM. Arrows indicate mitochondria; Scale bars, $1 \mu \mathrm{m}$.

The Expression Level of p-Akt

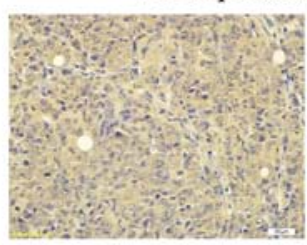

Control

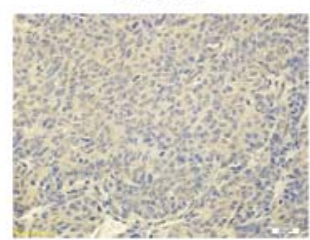

Emodin

(A)

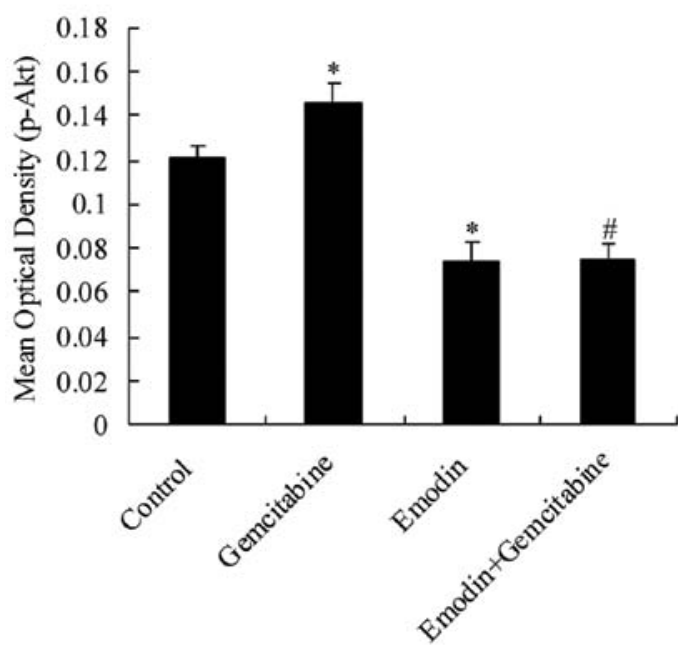

(B)

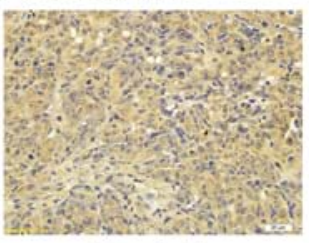

Gemcitabine

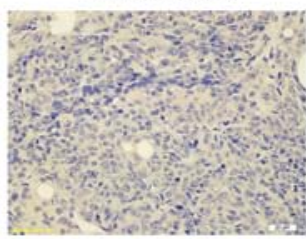

Emodin+Gemcitabine
phospho-Akt (Ser473)

$\beta$-actin

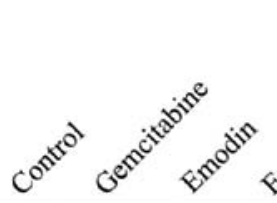

$=-$

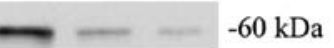

(C)

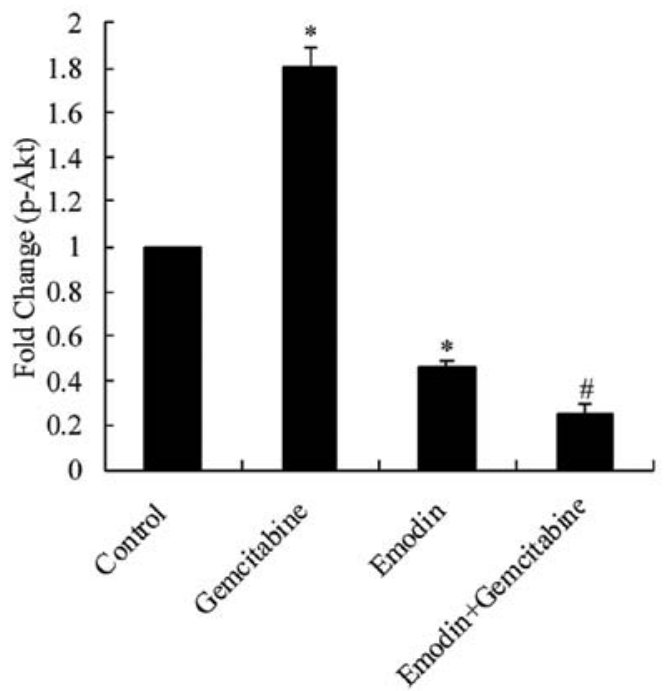

(D)

Figure 3. Combination treatment reduced Akt activation. One week after the final injection (day 37), the expression level of p-Akt was assessed by immunohistochemistry (A) and Western blotting (C). Quantified data are presented in (B) and (D). ${ }^{*} \mathrm{P}<0.01$ compared with sodium chloride treatment; ${ }^{*} \mathrm{P}<0.01$ compared to sodium chloride or gemcitabine treatment $(\mathrm{x} 400)$. 
Tumor Tissue

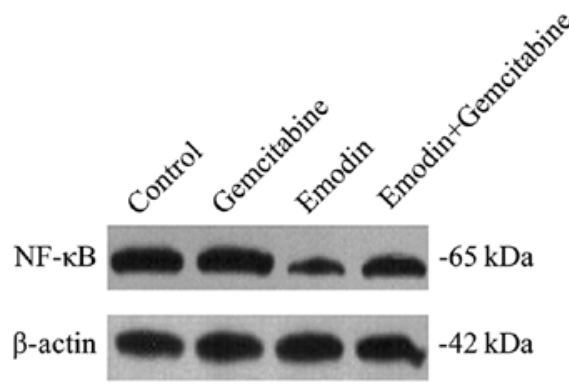

(A)

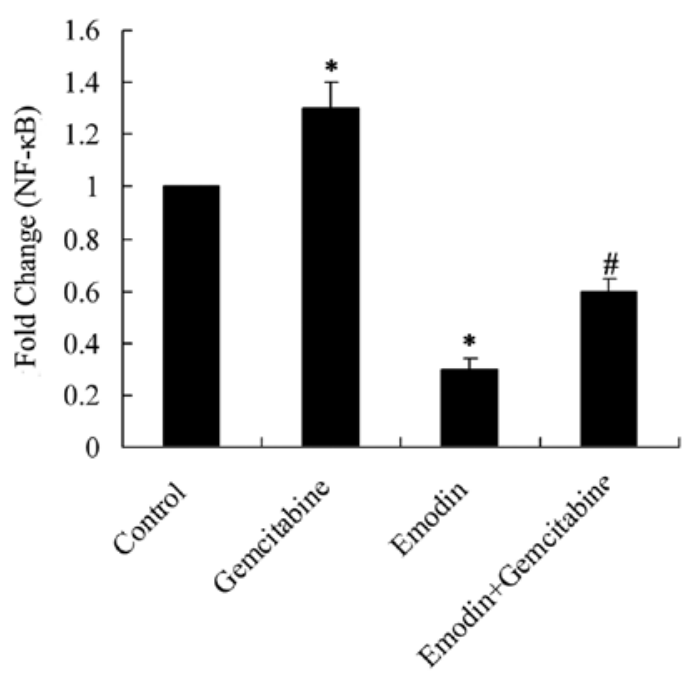

(C)

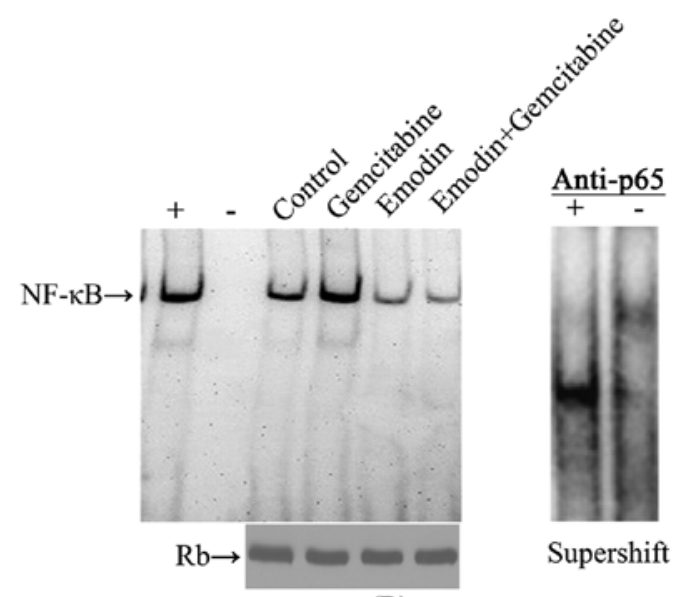

(B)

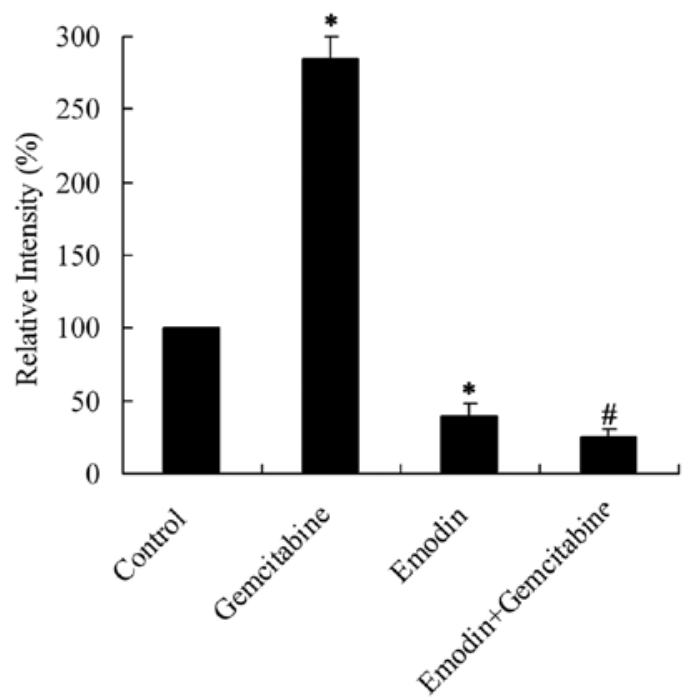

(D)

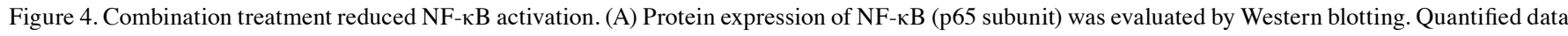
are presented in (B). (C) NF- $\mathrm{BB}$ DNA binding activity was determined by EMSA and supershift experiment was done. +, positive control; -, negative control. Quantified data are presented in (D). " $\mathrm{P}<0.01$ compared with sodium chloride treatment; ${ }^{\#} \mathrm{P}<0.01$ compared to sodium chloride or gemcitabine treatment.

we investigated the levels of p-Akt (Ser473) in different experimental groups. Immunohistochemical and immunoblot analysis for p-Akt revealed that gemcitabine treatment dramatically up-regulated the expression of p-Akt, whereas the addition of emodin significantly reduced the p-Akt level (Fig. 3A and C). Notably, combination therapy of emodin plus gemcitabine downregulated the p-Akt level as compared to monotherapy $(\mathrm{P}<0.01$, Fig. 3B and $\mathrm{D})$. These data provided insight into mechanistic basis on which emodin enhanced the effect of tumor cell apoptosis induced by gemcitabine.

Combination treatment reduced $N F-\kappa B$ activation. The transcription factor NF- $\kappa \mathrm{B}$ is an inhibitor of programmed cell death that acts as an anti-apoptotic factor in tumor cells (22). To determine whether combination treatment with emodin and gemcitabine has any effect on $\mathrm{NF}-\kappa \mathrm{B}$ activation, Western blotting using an anti-NF- $\mathrm{B}$ (p65 subunit) antibody was performed. Elevated expression of $\mathrm{NF}-\kappa \mathrm{B}$ (p65 subunit) protein was found in the gemcitabine monotherapy group, whereas emodin or emodin plus gemcitabine combination treatment significantly decreased $\mathrm{NF}-\kappa \mathrm{B}$ activation (Fig. 4A and B). Furthermore, NF- $\kappa$ B DNA binding activity was evaluated by EMSA. Equal protein loading was ensured by immunoblotting $10 \mu \mathrm{g}$ of nuclear protein with anti-retinoblastoma antibody. Consistent with immunoblotting results, emodin or emodin plus gemcitabine combination treatment decreased $\mathrm{NF}-\kappa \mathrm{B}$ binding activity (Fig. 4C and D), which was further confirmed by supershift experiment.

Combination treatment reduced the Bcl-2/Bax ratio, and promoted caspase- 3 activation and $C y t C$ release. Decreased $\mathrm{Bcl}-2 / \mathrm{Bax}$ ratio, activation of caspase- 9 and -3 , and $\mathrm{CytC}$ release are recognized as key events in the apoptotic pathway, and the pro-survival protein $\mathrm{Bcl}-2$ is reported to be a direct transcriptional target of NF- $\kappa \mathrm{B}$ (23). To further clarify the mechanism by which emodin and gemcitabine combination treatment promoted 

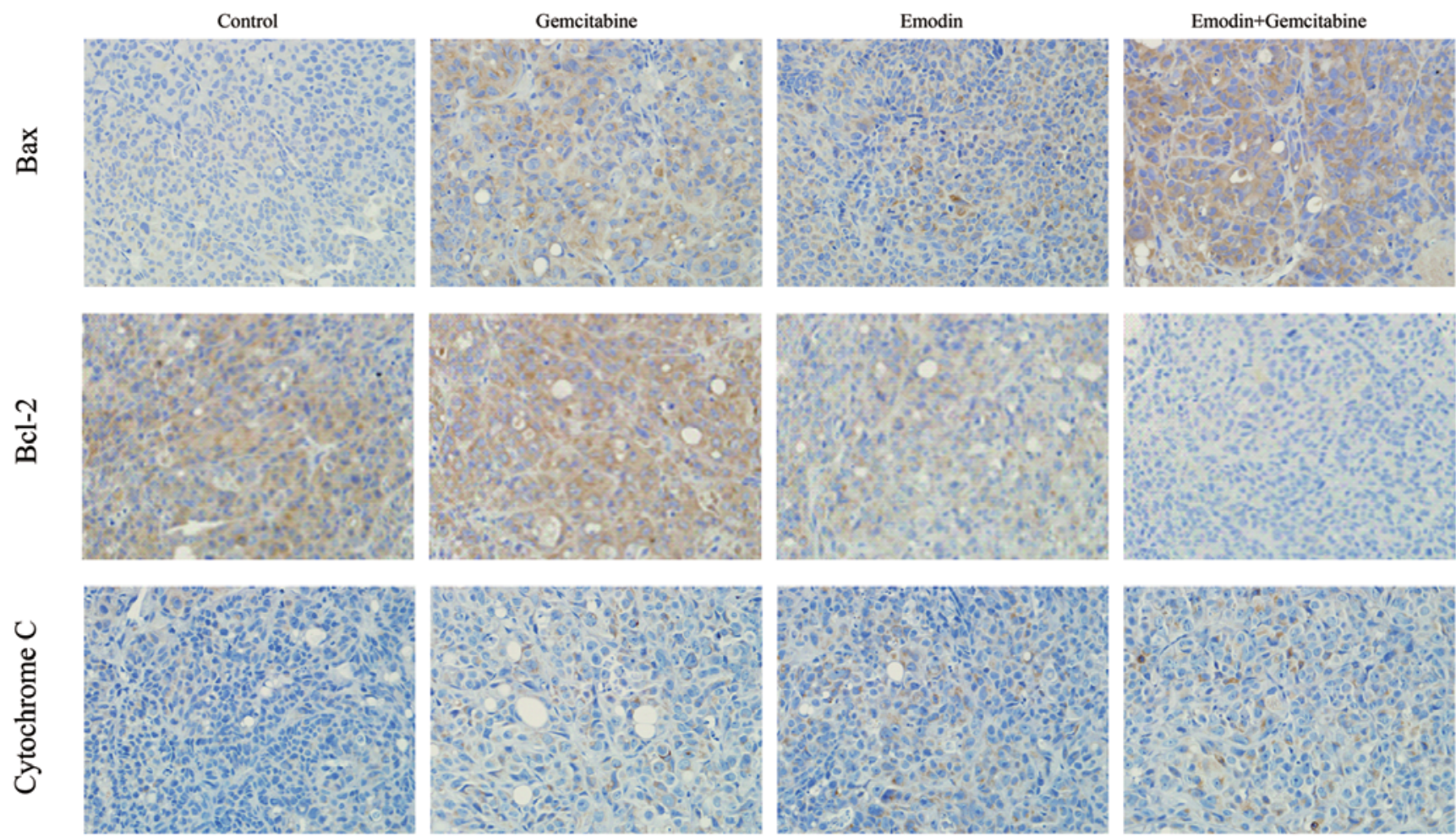

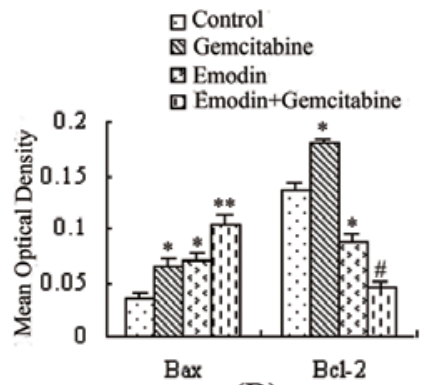

(B)

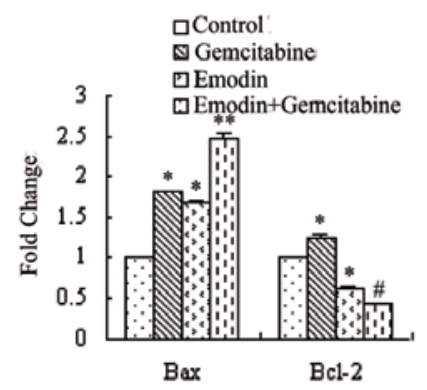

(F)
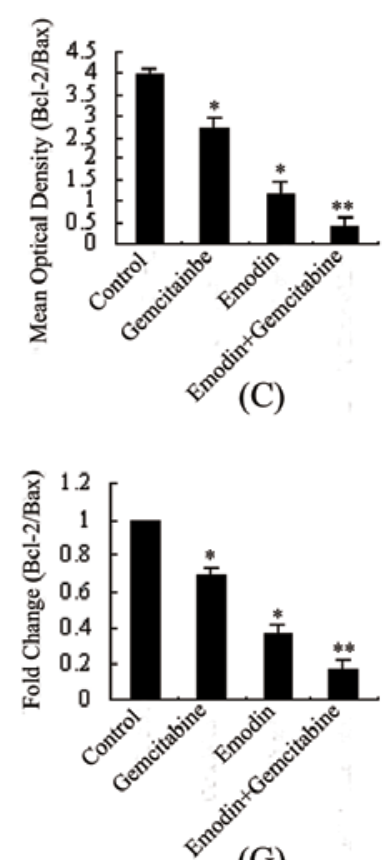

(G)

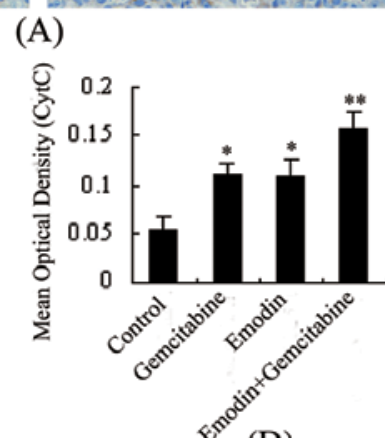

(D)

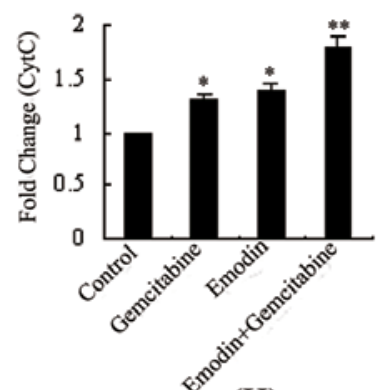

(H)

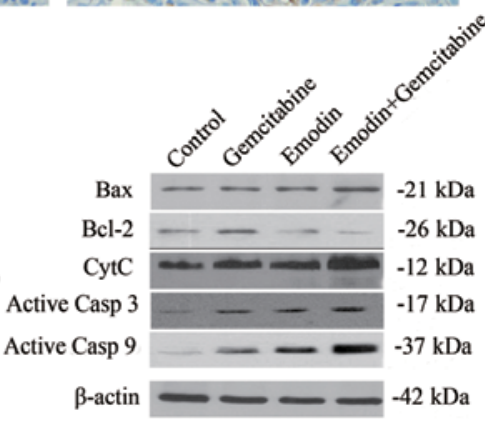

(E)

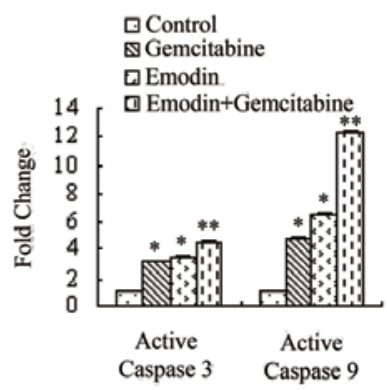

(I)

Figure 5. Combination treatment reduced the Bcl-2/Bax ratio, and promoted CytC release and caspase-9 and -3 activation. One week after the final injection (day 37), protein expression of Bcl-2, Bax, and CytC were assessed by immunohistochemistry (A), and Bcl-2, Bax, CytC and active caspase- 9 and -3 were evaluated by Western blotting (E). (B-D) Quantification of data from immunohistochemistry. (F-I) Quantification of data from Western blotting. "P<0.01 compared with sodium chloride treatment; ${ }^{* *} \mathrm{P}<0.01$ compared with sodium chloride, emodin or gemcitabine treatment; ${ }^{*} \mathrm{P}<0.01$ compared to sodium chloride or gemcitabine treatment $(\mathrm{x} 400)$.

apoptotic cell death in tumor tissues, the expression of antiapoptotic protein Bcl-2, pro-apoptotic protein Bax, and CytC were assessed by immunohistochemistry and Western blot analysis. A reduced $\mathrm{Bcl}-2 / \mathrm{Bax}$ ratio, but enhanced CytC release were observed in emodin and gemcitabine combination group after drug injection ( $\mathrm{P}<0.01$, Fig. 5A-D). Importantly, combi- nation therapy further promoted the reduction of the Bcl-2/Bax ratio $(\mathrm{P}<0.01$ compared with monotherapy with either agent, Fig. 5C). The ratio of $\mathrm{Bcl}-2 / \mathrm{Bax}$ is essential for regulating $\mathrm{CytC}$ release from the mitochondria, as well as caspase- 9 and -3 cleavage. Immunohistochemical analysis confirmed elevated cytosolic CytC levels in the combination treatment group 


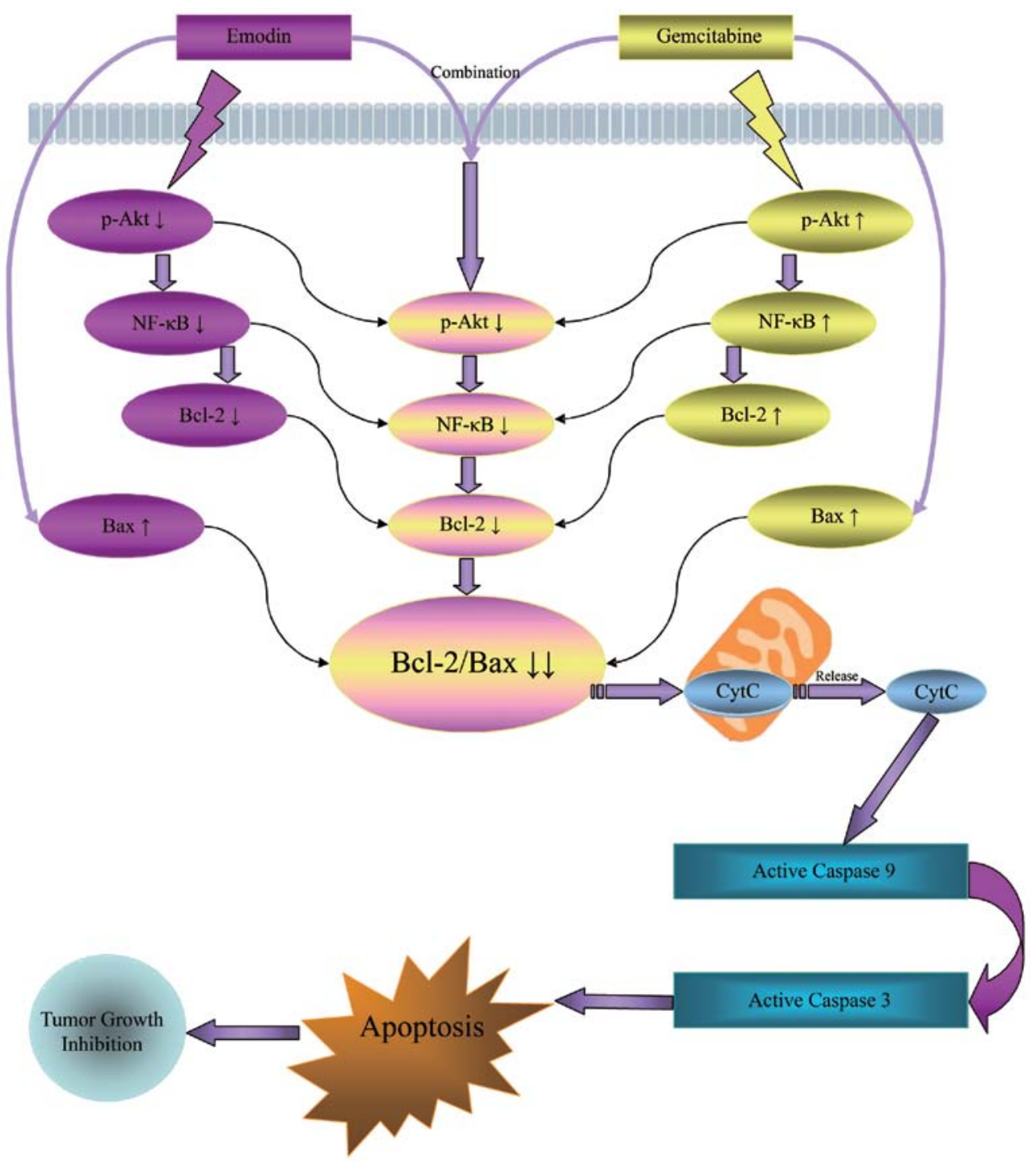

Figure 6. Scheme of anti-apoptosis pathways of emodin potentiating the antitumor activity of gemcitabine in pancreatic cancer.

$(\mathrm{P}<0.01$, Fig. 5D). Similar results were obtained from immunoblot analysis (Fig. 5E-H). Furthermore, immunoblot analysis indicated enhanced activation of caspase- 9 and -3 in the combination treatment group (Fig. 5I).

\section{Discussion}

Pancreatic cancer is one of the most common malignancies worldwide, with a relatively low 5-year survival rate (1). Doublet or triplet gemcitabine therapies in combination with 5-FU (8), oxaliplatin (9), cisplatin (10) or irinotecan (11) are known to provide improved pancreatic cancer survival outcomes. Although combination therapy extended the median survival of patients with advanced pancreatic cancer, the overall patient survival rate was still very low, due to the toxicity and side effects of these drugs. Although the results did not achieve statistical significance, a recent phase III trial of 195 patients with advanced adenocarcinoma of the pancreas found that the median overall survival and progression-free survival rates were more favorable for gemcitabine plus cisplatin combination therapy as compared with gemcitabine alone (10). Emodin, an active component from the rhizome of R. palmatum, has been reported to exhibit anti- tumor effects in various cancer cells (15). As a novel adjuvant chemotherapeutic drug, emodin has the advantages of low toxicity and low cost. In the current study, we investigated the effects of gemcitabine plus emodin combination therapy on the suppression of pancreatic tumor cell growth, and analyzed the potential mechanism involved in combination treatment-induced cancer cell apoptosis.

Three weeks after tumor cell inoculation, the mice received intraperitoneal injections of emodin, gemcitabine, emodin plus gemcitabine, or sodium chloride. Note that one week after the final injection, the average body weight of mice in the combination treatment group was significantly higher than that of the gemcitabine treatment group, whereas the average tumor size in the combination therapy group was dramatically smaller than monotherapy with either agent. These results demonstrate that a reduced dose of gemcitabine in combination with emodin exhibits decreased drug toxicity but enhances suppression of tumor growth.

Abnormal proliferation of tumor cells is usually associated with decreased apoptosis. Thus, promoting sensitivity to anti-cancer drugs and inducing apoptotic cell death is a critical chemotherapeutic strategy for patients with malignancies. 
Emerging studies suggest that emodin opposes tumor growth by triggering apoptosis $(16,19,24)$. Indeed, as revealed by TUNELstaining and TEM analysis, emodin or emodin plus gemcitabine combination treatment promoted apoptotic cell death in pancreatic tumors, and mitochondria appear to be the primary target of this therapy.

Akt, the downstream target of PI3K, is an essential cytoplasmic signal transduction protein which regulates cell apoptosis/survival. In physiological conditions, Akt is in inactive status in the cytoplasm. When exposed to certain stimuli, the T308 and S473 sites of Akt could be phosphorylated, which finally regulates cell apoptosis/survival. It has been reported that emodin could inhibit the activation of Akt and promote apoptosis of leukemia HL-60 cells (25). Olsen et al also showed that emodin can inhibit the activity of Akt in cervical cancer cells (26). Accumulating studies have indicated that inhibition of Akt can enhance the activity of gemcitabine chemotherapy in pancreatic cancer (27-29). Here, we found that emodin alone or combined with gemcitabine decreased the phospho-Akt (Ser473) levels in pancreatic tumor tissues of SW1990-inoculated nude mice, implying that emodin may also potentiate the antitumor activity of gemcitabine through inhibiting Akt activation. Fahy et al showed that Akt inhibition sensitized pancreatic cancer cells to the apoptotic effect of gemcitabine by suppressing the activity of $\mathrm{NF}-\kappa \mathrm{B}$ and reducing the Bcl-2/Bax ratio in pancreatic cancer cell line MIA-PaCa-2 (30). Consistent with a previous report, a remarkable deactivation of $\mathrm{NF}-\kappa \mathrm{B}$ and decrease in the $\mathrm{Bcl}-2 / \mathrm{Bax}$ ratio in tumor tissues was detected in emodin therapy group and in emodin plus gemcitabine combination group.

Activation of the transcription factor $\mathrm{NF}-\kappa \mathrm{B}$ after gemcitabine treatment has been found in studies of pancreatic cancer $(31,32)$. Consistent with these results, our study demonstrated that gemcitabine alone elevated $\mathrm{NF}-\kappa \mathrm{B}$ activation. As the activation of $\mathrm{NF}-\kappa \mathrm{B}$ may induce resistance of pancreatic cancer to gemcitabine treatment (33), it is possible that drug resistance occurred during monotherapy with gemcitabine. In contrast, emodin purportedly inhibits intracellular NF- $\mathrm{B}$ activity $(34,35)$. Here, both emodin alone and gemcitabine in combination with emodin significantly reduced NF- $\kappa \mathrm{B}$ (p65 subunit) as well as NF- $\mathrm{B}$ DNA binding activity, suggesting that emodin can potentiate the antitumor activity of gemcitabine via suppressing NF- $\mathrm{kB}$ activation, which is possibly associated with deactivation of Akt.

Since the pro-survival protein $\mathrm{Bcl}-2$ is reported to be a direct transcriptional target of $\mathrm{NF}-\kappa \mathrm{B}(23,33)$ and activated Akt regulates NF- $\mathrm{NB}$ activation (36) while inhibition of Akt can decrease the activity of NF- $\mathrm{BB}(30)$, as shown in our results, it is possible that $\mathrm{NF}-\kappa \mathrm{B}$ is an essential therapeutic target after emodin inhibits Akt activation, thus leading to decreased expression of $\mathrm{Bcl}-2$ in tumor tissues upregulated by gemcitabine as revealed in our results. Previous studies have shown that emodin down-regulates Bcl-2 expression, upregulates Bax expression, and triggers $\mathrm{CytC}$ release, subsequently activating caspase- $2,-9$, and -3 , and inducing apoptosis (18). The balance of pro-apoptotic and antiapoptotic proteins, such as the Bcl-2/Bax ratio, is essential in cell apoptosis, and disruption of this balance sensitizes cells to the apoptotic effects of chemotherapy. Gemcitabine has been shown to increase Bcl-2 expression due to NF- $\kappa \mathrm{B}$ activation (33) and resistance of pancreatic cancer to gemcitabine treatment is also known to be associated with changes in Bax expression (37). In this study, we found that emodin upregulated Bax expression and depressed gemcitabine-induced Bcl-2 upregulation in tumor tissues, and we observed a remarkable decrease in the Bcl-2/Bax ratio after emodin plus gemcitabine combination treatment, as revealed by immunohistochemical analysis and Western blotting. What's more, gemcitabine both upregulated Bax and Bcl-2, but downregulated $\mathrm{Bcl}-2 / \mathrm{Bax}$ ratio, thus induced apoptosis. Furthermore, consistent with a previous report (19), combination treatment elevated cytosolic CytC levels and activated caspase-9 and -3 protein expression compared with monotherapy with either agent, suggesting that gemcitabine plus emodin leads to activation of a mitochondrial-dependent apoptotic signaling pathway.

Collectively, the present study demonstrated that combination treatment consisting of gemcitabine and emodin efficiently suppressed tumor growth in SW1990 pancreatic tumor cellinoculated mice compared to mice receiving monotherapy with either agent. Combination treatment promoted apoptotic cell death in pancreatic tumor cells through deactivation of Akt and then $\mathrm{NF}-\kappa \mathrm{B}$, and subsequently reduced the $\mathrm{Bcl}-2 / \mathrm{Bax}$ ratio, enhanced CytC release and promoted caspase- 9 and -3 activation (Fig. 6). Hence, these results suggest that emodin potentiates the antitumor activity of gemcitabine via deactivation of Akt and then NF- $\mathrm{BB}$, and finally activation of mitochondria-dependent apoptotic pathway, and that combination therapy may provide new alternatives for the clinical treatment of patients with pancreatic tumors. However, the dose of each agent in this combination therapy must be optimized prior to clinical application.

\section{Acknowledgements}

This work was supported by grants from the Natural Science Foundation of Zhejiang Province, China (Grant No. Y2080708) and The Administration of Traditional Chinese Medicine of Zhengjing Province, China (Grant No. 2011ZZ010).

\section{References}

1. Jemal A, Siegel R, Ward E, Hao Y, Xu J, Murray T and Thun MJ: Cancer statistics, 2008. CA Cancer J Clin 58: 71-96, 2008.

2. Thomas A, Dajani K, Neoptolemos JP and Ghaneh P: Adjuvant therapy in pancreatic cancer. Dig Dis 28: 684-692, 2010.

3. Huguet F, Orthuon A, Touboul E, Marseguerra R and Mornex F: Pancreatic cancer. Cancer Radiother 14 (Suppl. 1): S94-S102, 2010.

4. Hilbig A and Oettle $\mathrm{H}$ : Adjuvant therapy of pancreatic cancer. Expert Rev Anticancer Ther 10: 485-491, 2010.

5. Shore S, Raraty MG, Ghaneh P and Neoptolemos JP: Review article: chemotherapy for pancreatic cancer. Aliment Pharmacol Ther 18: 1049-1069, 2003.

6. Carmichael J, Fink U, Russell RC, Spittle MF, Harris AL, Spiessi G and Blatter J: Phase II study of gemcitabine in patients with advanced pancreatic cancer. Br J Cancer 73: 101-105, 1996.

7. Burris HA III, Moore MJ, Andersen J, et al: Improvements in survival and clinical benefit with gemcitabine as first-line therapy for patients with advanced pancreas cancer: a randomized trial. J Clin Oncol 15: 2403-2413, 1997.

8. Berlin JD, Catalano P, Thomas JP, Kugler JW, Haller DG and Benson AB III: Phase III study of gemcitabine in combination with fluorouracil versus gemcitabine alone in patients with advanced pancreatic carcinoma: Eastern Cooperative Oncology Group Trial E2297. J Clin Oncol 20: 3270-3275, 2002.

9. Louvet C, Labianca R, Hammel P, et al: Gemcitabine in combination with oxaliplatin compared with gemcitabine alone in locally advanced or metastatic pancreatic cancer: results of a GERCOR and GISCAD phase III trial. J Clin Oncol 23: 3509-3516, 2005. 
10. Heinemann V, Quietzsch D, GieselerF, et al: Randomized phase II trial of gemcitabine plus cisplatin compared with gemcitabine alone in advanced pancreatic cancer. J Clin Oncol 24: 3946-3952, 2006.

11. Rocha Lima CM, Savarese D, Bruckner H, et al: Irinotecan plus gemcitabine induces both radiographic and CA 19-9 tumor marker responses in patients with previously untreated advanced pancreatic cancer. J Clin Oncol 20: 1182-1191, 2002.

12. Kullmann F, Hollerbach S, Dollinger MM, et al: Cetuximab plus gemcitabine/oxaliplatin (GEMOXCET) in first-line metastatic pancreatic cancer: a multicentre phase II study. Br J Cancer 100: 1032-1036, 2009.

13. Sato M, Maulik G, Bagchi D and Das DK: Myocardial protection by protykin, a novel extract of trans-resveratrol and emodin. Free Radic Res 32: 135-144, 2000.

14. Lin SZ, Chen KJ, Tong HF, Jing H, Li H and Zheng SS: Emodin attenuates acute rejection of liver allografts by inhibiting hepatocellular apoptosis and modulating the Th1/Th2 balance in rats. Clin Exp Pharmacol Physiol 37: 790-794, 2010.

15. Liu A, Chen H, Wei W, et al: Antiproliferative and antimetastatic effects of emodin on human pancreatic cancer. Oncol Rep 26 81-89, 2011.

16. Chen H, Wei W, Guo Y, et al: Enhanced effect of gemcitabine by emodin against pancreatic cancer in vivo via cytochrome $\mathrm{C}$ regulated apoptosis. Oncol Rep 25: 1253-1261, 2011.

17. Chen YC, Shen SC, Lee WR, Hsu FL, Lin HY, Ko CH and Tseng SW: Emodin induces apoptosis in human promyeloleukemic HL-60 cells accompanied by activation of caspase 3 cascade but independent of reactive oxygen species production. Biochem Pharmacol 64: 1713-1724, 2002.

18. Su YT, Chang HL, Shyue SK and Hsu SL: Emodin induces apoptosis in human lung adenocarcinoma cells through a reactive oxygen species-dependent mitochondrial signaling pathway. Biochem Pharmacol 70: 229-241, 2005.

19. Guo Q, Chen Y, Zhang B, Kang M, Xie Q and Wu Y: Potentiation of the effect of gemcitabine by emodin in pancreatic cancer is associated with survivin inhibition. Biochem Pharmacol 77: 1674-1683, 2009.

20. Khanbolooki S, Nawrocki ST, Arumugam T, et al: Nuclear factorkappaB maintains TRAIL resistance in human pancreatic cancer cells. Mol Cancer Ther 5: 2251-2260, 2006

21. Vandermoere F, El Yazidi-Belkoura I, Adriaenssens E, Lemoine J and Hondermarck $\mathrm{H}$ : The antiapoptotic effect of fibroblast growth factor-2 is mediated through nuclear factor-kappaB activation induced via interaction between Akt and IkappaB kinase-beta in breast cancer cells. Oncogene 24: 5482-5491, 2005.

22. Karin M, Cao Y, Greten FR and Li ZW: NF-kappaB in cancer: from innocent bystander to major culprit. Nat Rev Cancer 2 : 301-310, 2002

23. Zong WX, Edelstein LC, Chen C, Bash J and Gelinas C: The prosurvival Bcl-2 homolog $\mathrm{Bfl}-1 / \mathrm{A} 1$ is a direct transcriptional target of NF-kappaB that blocks TNFalpha-induced apoptosis. Genes Dev 13: 382-387, 1999.
24. Cai J, Razzak A, Hering J, Saed A, Babcock TA, Helton S and Espat NJ: Feasibility evaluation of emodin (rhubarb extract) as an inhibitor of pancreatic cancer cell proliferation in vitro. JPEN J Parenter Enteral Nutr 32: 190-196, 2008.

25. Zheng HY, Hu JD, Zheng ZH, et al: [Emodin induces leukemic HL-60 cells apoptosis probably by inhibiting Akt signal pathway]. Yao Xue Xue Bao 42: 1142-1146, 2007.

26. Olsen BB, Bjorling-Poulsen M and Guerra B: Emodin negatively affects the phosphoinositide 3-kinase/AKT signalling pathway: a study on its mechanism of action. Int J Biochem Cell Biol 39: 227-237, 2007.

27. Liu D, Zhang Y, Dang C, Ma Q, Lee W and Chen W: siRNA directed against TrkA sensitizes human pancreatic cancer cells to apoptosis induced by gemcitabine through an inactivation of PI3K/Akt-dependent pathway. Oncol Rep 18: 673-677, 2007.

28. Yao J and Qian C: Inhibition of Notch3 enhances sensitivity to gemcitabine in pancreatic cancer through an inactivation of PI3K/ Akt-dependent pathway. Med Oncol 27: 1017-1022, 2010.

29. Zhang B, Shi ZL, Liu B, Yan XB, Feng J and Tao HM: Enhanced anticancer effect of gemcitabine by genistein in osteosarcoma: the role of Akt and nuclear factor-kappaB. Anticancer Drugs 21: 288-296, 2010.

30. Fahy BN, Schlieman M, Virudachalam S and Bold RJ: AKT inhibition is associated with chemosensitisation in the pancreatic cancer cell line MIA-PaCa-2. Br J Cancer 89: 391-397, 2003.

31. Lee SH, Ryu JK, Lee KY, et al: Enhanced anti-tumor effect of combination therapy with gemcitabine and apigenin in pancreatic cancer. Cancer Lett 259: 39-49, 2008.

32. Uwagawa T, Chiao PJ, Gocho T, Hirohara S, Misawa T and Yanaga K: Combination chemotherapy of nafamostat mesilate with gemcitabine for pancreatic cancer targeting NF-kappaB activation. Anticancer Res 29: 3173-3178, 2009.

33. Fahy BN, Schlieman MG, Virudachalam S and Bold RJ: Inhibition of AKT abrogates chemotherapy-induced NF-kappaB survival mechanisms: implications for therapy in pancreatic cancer. J Am Coll Surg 198: 591-599, 2004

34. Li HL, Chen HL, Li H, et al: Regulatory effects of emodin on NF-kappaB activation and inflammatory cytokine expression in RAW 264.7 macrophages. Int J Mol Med 16: 41-47, 2005.

35. Kuo TC, Yang JS, Lin MW, et al: Emodin has cytotoxic and protective effects in rat C6 glioma cells: roles of Mdr1a and nuclear factor kappaB in cell survival. J Pharmacol Exp Ther 330: 736-744, 2009

36. Jeong SJ, Pise-Masison CA, Radonovich MF, Park HU and Brady JN: Activated AKT regulates NF-kappaB activation, p53 inhibition and cell survival in HTLV-1-transformed cells. Oncogene 24: 6719-6728, 2005.

37. Schniewind B, Christgen M, Kurdow R, Haye S, Kremer B, Kalthoff $\mathrm{H}$ and Ungefroren $\mathrm{H}$ : Resistance of pancreatic cancer to gemcitabine treatment is dependent on mitochondria-mediated apoptosis. Int J Cancer 109: 182-188, 2004. 